$57 / 96 / \mathrm{EP}$

April 1996

\title{
Quantum Deformation Of Lattice Gauge Theory
}

\author{
D.V. Boulatov \\ International School for Advanced Studies (SISSA/ISAS) \\ via Beirut 2-4, I-34014 Trieste, Italy
}

\begin{abstract}
A quantum deformation of 3-dimensional lattice gauge theory is defined by applying the Reshetikhin-Turaev functor to a Heegaard diagram associated to a given cell complex. In the root-of-unity case, the construction is carried out with a modular Hopf algebra. In the topological (weak-coupling) limit, the gauge theory partition function gives a 3fold invariant, coinciding in the simplicial case with the Turaev-Viro one. We discuss bounded manifolds as well as links in manifolds. By a dimensional reduction, we obtain a $q$-deformed gauge theory on Riemann surfaces and find a connection with the algebraic Alekseev-Grosse-Schomerus approach.
\end{abstract}




\section{Introduction}

The lattice regularization of non-abelian gauge theory (LGT) proposed by K.Wilson in 1975 [1] plays a fundamental role of a non-perturbative definition of QCD. The main principle has been to give up the Poincaré invariance of the theory and preserve the local gauge symmetry as more fundamental. In the weak coupling regime, the broken translational and rotational invariance restores dynamically. If the gauge coupling is strictly equal to 0 , the gauge strength tensor vanishes identically and the theory becomes topological: one can take any finite lattice from a given equivalence class without changing the content of the model.

The lattice formulation extends in a natural way the set of acceptable gauge groups for all compact groups while the continuous one is based on the notion of the Lie algebra thus excluding finite groups, for example. After the theory of quantum groups had appeared as a distinct mathematical subject [2, 3], the natural question arose whether the notion of gauge symmetry could be extended to incorporate quantum groups as well. This problem has not been only of academic interest. As was noticed in Ref. [U], the Ponzano-Regge model [5] (which coincides with the classical $(q \rightarrow 1)$ limit of the Turaev-Viro construction [6]) can be represented as LGT defined on lattices dual to simplicial complexes. Then it was natural to assume that the Turaev-Viro invariant could be related to a kind of LGT built on quantum group symmetry (for the sake of brevity, we shall call it $\mathrm{qQCD}_{3}$ ). This program was carried out in Ref. [7] explicitely (see also [8]). Technical difficulties originating from a complicated structure of the representation ring of $S L_{q}(2)$ at a root of

unity, $q=e^{i \frac{2 \pi}{k+2}}$, was avoided in [0] by establishing a direct connection with the ribbon graph invariants of Reshetikhin and Turaev [9]. The gauge invariance is implicit in this formulation (however, it does not mean that the model does not enjoy it). On the other hand, relative simplicity makes this explicit representation very convenient.

The classical $(q \rightarrow 1)$ limit of the Turaev-Viro construction was discovered long ago by Ponzano and Regge in the framework of Regge calculus [5]. They argued that it can be regarded as a discretization of $3 \mathrm{~d}$ gravity with the Einstein-Hilbert action. On the other hand, the Turaev-Viro invariant is related to Witten's Chern-Simons invariant [10]. Witten has shown that, with the $\operatorname{ISO}(2,1)$ gauge group, the latter is connected with $3 \mathrm{~d}$ quantum gravity. In the Euclidean regime for a negative cosmological constant, the gauge group becomes isomorphic to $S O(4)=S U(2) \times S U(2)$. It means that qQCD 3 possessing $S L_{q}(2)$ gauge symmetry is interesting from the physical point of view (an exposition of the subject can be found in Ref. [8] ).

The structure of the answers for the partition function suggests that, in 2 dimensions, the corresponding q-deformed LGT $\left(\mathrm{qQCD}_{2}\right)$ is related to the topological $(G / G)_{k}$ coset model (as was argued in Ref. [11] these two models are in some sense dual to each other).

An alternative purely algebraic approach to $\mathrm{qQCD}_{2}$ was put forward in Ref. [12, 13, 14]. The starting point for them was a Poisson bracket on $2 \mathrm{~d}$ lattice connections proposed by 
Fock and Rosly [15.

In 2 dimensions there is a cyclic order of links incident to a vertex. Demanding that variables performing gauge rotations at each vertex form a quasi-triangular Hopf algebra, Alekseev, Grosse and Schomerus have deduced an algebra of gauge fields. In contrast to this situation, in 3 dimensions there is a natural cyclic order of faces sharing the same link of a lattice, which suggests that one should start with gauge fields forming a Hopf algebra while gauge transformations are interpreted as changes of bases these fields act on. As in the quantum case there is no group manifold behind the construction, gauge fields and

gauge transformations have clearly the different statuses. One could say that this is one more occurrence of the principle "Quantization removes degenerations". Descending from 3 to 2 dimensions, one finds a model which seems, at first sight, to be different from the AGS one. However, as we shall show they are locally equivalent.

The outline of the paper is the following. Chapter 2 is devoted to the construction of $q$-deformed LGT in three dimensions. In Section 2.1 we introduce classical LGT and make some general remarks on its quantum deformation.

In Section 2.2 we collect facts from 3-manifold topology which are used in the sequel.

In Section 2.3 we introduce the Reshetikhin-Turaev functor in the form adopted for our purposes.

In Section 2.4 we define $\mathrm{qQCD}_{3}$ functor and discuss the notion of gauge invariance within our framework.

In Section 2.5 we introduce the $\mathrm{qQCD}_{3}$ partition function in the case of $\mathcal{U}_{q}(s u(n))$ gauge group.

Section 2.6 is devoted to the root-of-unity case: $\mathcal{U}_{q}(\operatorname{sl}(n, R)), q^{\ell}=1$.

In Section 2.7 we prove that the weak-coupling partition function introduced in the previous section is a topological invariant.

In Section 2.8 we discuss the case of bounded manifolds and shortly outline the introduction of Wilson loop averages in our model.

Chapter 3 is devoted to the 2-dimensional case. Here we derive Verlinde's formula and discuss a connection between our approach and the AGS algebra.

We conclude with a few general remarks.

\section{$2 \mathrm{qQCD}_{3}$}

\subsection{Formulation of the problem}

To introduce lattice gauge theory, one needs a cell decomposition of a manifold (in physicist usage, a lattice). A gauge field is a map from a set of oriented edges to a compact group: $\ell \mapsto g_{\ell} \in G$. A change of an orientation corresponds to the conjugation: $g_{\ell} \rightarrow g_{\ell}^{-1}$.

One attaches to every vertex a $G$-module (usually the regular representation). Gauge 
transformations rotate bases of the modules independently at each vertex. The gauge field is interpreted as performing a parallel transport between vertices, thus relating bases at adjacent ones.

If an oriented link, $k$, connects vertices $v_{2}$ and $v_{1}$, the gauge transformation of the group element $g_{k}$ is

$$
g_{k} \rightarrow h_{v_{1}} g_{k} h_{v_{2}}^{-1}
$$

A holonomy associated with a path $\{L\}$ in the lattice is an ordered product of gauge field elements along $\{L\}$ :

$$
h_{L}=\prod_{k \in L} g_{k}^{\epsilon_{k}}
$$

where $\epsilon_{k}=+1$, if the $k$-th edge is directed along the path, and $\epsilon_{k}=-1$, if their directions are opposite.

Gauge invariant quantities are those taking values in the set of conjugacy classes of $G$. A trace of the holonomy along a closed loop in any representation of $G$ is an example of such an invariant.

The Boltzmann weights are functions of holonomies along boundaries, $\partial f$, of faces, $f$. One of the standard choices is the so-called group heat kernel

$$
W_{\beta}\left(h_{\partial f}\right)=\sum_{R} d_{R} \chi_{R}\left(h_{\partial f}\right) e^{-\beta C_{R}}
$$

In eq. (2.3), $\sum_{R}$ is the sum over all finite dimensional irreps of a gauge group $G ; \chi_{R}(x)$ is the character of an irrep $R ; d_{R}=\chi_{R}(I)$ is its dimension; $C_{R}$ is a second Casimir eigenvalue; $\beta$ is a real parameter called a coupling constant. The construction makes sense for compact groups whose unitary finite dimensional irreps span the regular representation.

The choice (2.3) ensures that $W_{\beta}\left(h_{\partial f}\right)$ becomes the group $\delta$-function in the weak coupling limit, $\beta \rightarrow 0: W_{0}\left(h_{\partial f}\right)=\delta\left(h_{\partial f}, I\right)$. We shall call this limit topological.

The partition function is defined as the integral of the product of the Boltzmann weights over all faces:

$$
\mathcal{Z}_{\beta}=\int_{G} \prod_{\ell} d g_{\ell} \prod_{f} W_{\beta}\left(\prod_{k \in \partial f} g_{k}^{\epsilon_{k}}\right)
$$

where $d g_{\ell}$ is the Haar measure on the group $G$, and the product $\prod_{\ell}$ goes over all edges.

If the term "q-deformed" is to mean that gauge variables take values in a quantum group, any presentation of the model should be reducible to a form where the variables are represented in a standard fashion as matrices of non-commutative elements. 
The simplest and most famous example is $S L_{q}(2)$, which can be introduced as the set of matrices

$$
g=\left(\begin{array}{ll}
a & b \\
c & d
\end{array}\right)
$$

the entries of which obey the commutation relations

$$
\begin{array}{lll}
b a=q a b \quad d b=q b d & c b=b c \\
c a=q a c \quad d c=q c d & d a-a d=\left(q-q^{-1}\right) b c \\
a d-q^{-1} b c=1 &
\end{array}
$$

The relations (2.6) imply the existence of the $R$-matrix

$$
R=\left(\begin{array}{cccc}
q & 0 & 0 & 0 \\
0 & 1 & 0 & 0 \\
0 & q-q^{-1} & 1 & 0 \\
0 & 0 & 0 & q
\end{array}\right)
$$

and the $R T T=T T R$ equation

$$
(g \otimes 1)(1 \otimes g) R=R(1 \otimes g)(g \otimes 1)
$$

The $R$-matrix obeys the quantum Yang-Baxter equation

$$
R_{12} R_{13} R_{23}=R_{23} R_{13} R_{12}
$$

Indices show at which positions in the tensor cube of representation spaces, $\stackrel{1}{V} \otimes \stackrel{2}{V} \otimes \stackrel{3}{V}$, acts the $R$-matrix.

$S L_{q}(2)$ has two real forms: $S U_{q}(2)$, for real $q$, and $S L_{q}(2, R)$, for $|q|=1$.

The matrices can be multiplied. If entries of both $g$ and $h$ obey Eq. (2.6) and are mutually commutative, the entries of the product $g h$ obey (2.6) as well. Therefore, matrices on different links of a lattice have to co-commute with one another in the tensor product.

The algebra of matrices (2.5) naturally extends to the quasi-triangular Hopf algebra $\mathcal{F}_{q}(S L(2))$ of quantized functions on $S L(2)$. Owing to the famous duality, its basis is provided by the matrix elements of finite-dimensional irreducible representations of the quantized universal envelopping (QUE) algebra $\mathcal{U}_{q}(s l(2))$. Therefore, to construct qQCD, we have at hands co-multiplication, $R$-matrix, antipode and Clebsch-Gordan coefficients (CGC).

The q-deformation of Eq. (2.4) is roughly speaking a way to write it down in terms of elements of the Hopf algebra. A priori, it is not unique. The guiding principle here can be to identify any transformation of the construction with some isometry of a base cell complex in a self-consistent way. Then all algebraic manipulations become geometrically 
meaningful. It is close in spirit to the Reshetikhin-Turaev functor from the category of ribbon tangles to the modular Hopf algebras [9]. Our presentation of $\mathrm{qQCD}_{3}$ is in many respects inspired by their work. To describe it, we need to look at LGT from a bit more general than usual point of view.

\subsection{Topological background}

For reader's convenience we collect in this section some definitions which we shall use in the sequel.

A $k$-cell is a polyhedron homeomorphic to a $k$-dimensional ball.

A cell complex is a union of a finite number of cells such that an intersection of any 2 $k$-cells is either empty or a finite number of less dimensional cells.

A cell complex can be obtained starting with a finite set of points by attaching subsequently cells of higher dimensions, any cell being attached to a finite number of lower dimensional cells.

A union of all cells of dimension $\leq n$ is called an $n$-skeleton.

A cell complex is a manifold if and only if the neighbourhood of each vertex is a spherical ball.

A complex is called simplicial if all cells are simplexes (i.e., points, links, triangles, tetrahedra, etc.).

Physicists usually mean by a lattice a cell complex such that an intersection of any two $k$-cells either empty or consists of only one entire less dimensional cell. We adopt this notion. Simplicial complexes are lattices by definition.

A dual complex, $\widetilde{C}$, is constructed by putting into correspondence its $k$-cells to cells of $C$ having complimentary dimensions, $n-k$.

To introduce LGT, we need a presentation of a cell complex, i.e., an effective way to describe it unambiguously. In the classical case, one needs to know only a 2-skeleton of a complex.

From the topological point of view, the construction of LGT described at the beginning of the previous section is reminiscent of the definition of $H^{1}(C, G)$, the non-commutative first cohomology of $C$ with coefficients in $G$. In the topological limit, all holonomies along contractible loops vanish and gauge fields obey the defining relations of $\pi_{1}(C)$. Therefore, being properly normalized, the partition function $\mathcal{Z}_{0}$ counts the number of conjugacy classes of injective homomorphisms from $\pi_{1}(C)$ into a gauge group $G$ :

$$
\mathcal{Z}_{0}=\left|\operatorname{Hom}\left(\pi_{1}(C), G\right) / G\right|
$$

Of course, it makes sense only if $G$ is finite. If $G$ is a Lie group, one speaks about 
a moduli space of flat $G$-connections, which is defined as a set of fields modulo gauge transformations:

$$
\mathcal{M}_{G}:=\left\{\operatorname{Hom}\left(\pi_{1}(C), G\right) / G\right\}
$$

It is easy to see that classical topological LGT is completely determined by a homotopy type of a complex. The construction of $\mathrm{qQCD}_{3}$ requires a more precise presentation of a complex.

It is known that any oriented 3-manifold can be obtained by gluing up two 3-dimensional handlebodies along their boundaries. This operation is the Heegaard splitting. The minimal genus of the handlebodies is called the Heegaard genus of the manifold.

We can obtain a Heegaard splitting for a given oriented manifold $M$ from its cellular decomposition, $C$, as follows. We take a tubular neighbourhood, $H$, of the 1 -skeleton of $C$. The complement of $H$ in $M, \widetilde{H}=M \backslash H$, can be regarded as a tubular neighbourhood of the 1-skeleton of the dual complex $\widetilde{C}$.

Every 1-cell $\sigma_{i}^{1} \in C$ determines a disk $D_{i} \subset H$ whose detachment distroys a handle of $H$. The boundaries of the disks $\partial D_{i} \subset \partial H$ give a system of cycles on the boundary, $\partial H$, of the handlebody $H$. We shall call them the $\alpha$-cycles: $\alpha_{i}:=\partial D_{i}$. Dual 1-cells $\widetilde{\sigma_{j}^{1}} \in \widetilde{C}$ determine analogously a system of $\widetilde{\alpha}$-cycles on the boundary, $\partial \widetilde{H}$, of $\widetilde{H}$. Images of the $\widetilde{\alpha}$-cycles on $\partial H$ produced by a gluing homomorphism $h$ are called the characteristic curves (or $\gamma$-cycles) of the Heegaard diagram and define the manifold $M=H \bigcup_{h} \widetilde{H}$ unambiguously.

Let us fix a number of the disks $\{\widehat{D}\} \subset\{D\}$ such that the detachment of them makes the handlebody connected and simply-connected (i.e., $H \backslash\{\widehat{D}\} \cong B^{3}$ ). We can put into correspondence a generator $a_{i}$ of the fundamental group to each disk $D_{i} \in\{\widehat{D}\}$. Defining relations are read off in an obvious way from a system of the characteristic curves $\{\gamma\}$. That is, if $\gamma_{j}$ intersects disks $D_{j_{1}}, D_{j_{2}}, \ldots, D_{j_{k}}$ subsequently, then the corresponding relator is $\Gamma_{j}=a_{j_{1}}^{\epsilon_{j_{1}}} a_{j_{2}}^{\epsilon_{j_{2}}} \ldots a_{j_{k}}^{\epsilon_{j_{k}}}$, where $\epsilon_{k}= \pm 1$ is the intersection number depending on a mutual orientation of $\gamma_{j}$ and the $k$-th disk at the intersection point. This set of relators is of course excessive. A minimal set can be fixed by choosing a number of $\widetilde{\alpha}$-cycles which span a disjoint collection of disks $\{\widehat{\widetilde{D}}\}$ in the complementary handlebody $\widetilde{H}=M \backslash H$ such that the detachment of all the disks from the set $\{\widehat{\widetilde{D}}\}$ makes $\widetilde{H}$ a 3 -ball: $\widetilde{H} \backslash\{\widehat{\widetilde{D}}\} \cong B^{3}$.

One can deform a Heegaard diagram by any 2-dimensional isomorphism of a boundary $\partial H$ which extends to the whole handlebody $H$. A set of generators for such isomorphisms is called in the literature the Suzuki moves (see, e.g., Refs. [16, 17] for an exposition accessible to a physicist).

It can be shown that any class of isotopic diffeomorphisms of a genus $g$ surface $M_{g}^{2}$ onto itself has a representative which can be constructed as a composition of the Dehn twists, $T_{\mu}^{\epsilon}$, where $\mu$ is one of the basic cycles on $M_{g}^{2}$ and $\epsilon= \pm 1$. One detaches from $M_{g}^{2}$ a thin neighborhood $U_{\mu} \cong S^{1} \times[0,1]$ of a cycle $\mu$ and then attaches it back after the full 
twist $U_{\mu} \rightarrow U_{\mu}: \varphi \times t \rightarrow(\varphi+2 \pi \epsilon t) \times t$ (where $t \in[0,1]$ and $\phi \in[0,2 \pi]$ parametrizes $S^{1}$ ).

In the sequel, we shall only need the following fact: all the Suzuki moves are combinations of Dehn twists on loops in $\partial H$ which bound disks $D \subset H$, except for the handle slide defined in the following way. Imagine solid handles attached to a surface of a spherical ball. One drags one end of a handle up, along and down another handle. As a result of this operation, an $\alpha$-cycle corresponding to the second handle slides around an $\alpha$-cycle corresponding to the first one. It can be described as a multiplication of loops on $\partial H$ defined in the standard fashion as in the definition of the fundamental group $\pi_{1}\left(M_{g}^{2}\right)$

The same operations can be applied to $\widetilde{H}$ as well.

It is a classical result that any two Heegaard diagrams representing the same manifold can be connected by a sequence of the following operations:

1. Dehn twists on loops contractible in $H$ or $\widetilde{H}$. They do not change a presentation of $\pi_{1}(C)$.

2. Cycle slide, which consists in the multiplication of a cycle by another one: $\gamma_{j} \rightarrow \gamma_{j} \gamma_{k}$. It means that a relator $\Gamma_{j}$ in a presentation of $\pi_{1}(C)$ is substituted by $\Gamma_{j} \Gamma_{k}$. The same operation applied to the $\alpha$-cycles, $\alpha_{j} \rightarrow \alpha_{j} \alpha_{k}$, corresponds to the change of generators of $\pi_{1}(C): a_{j}$ is substituted for $a_{j} a_{k}$.

3. Stabilization, which consists in adding a new handle to $H$ and extending a gluing diffeomorphism by the identity on its boundary. It means that one adds one characteristic curve and one $\alpha$-cycle to a Heegaard diagram or, equivalently, a new generator $a_{g+1}$ along with the trivial relation, $\Gamma_{g+1}=a_{g+1}=1$, to a presentation of $\pi_{1}(C)$.

It should be noted that an isotopy within a handlebody itself cannot necessarily take place for its embeddings in $R^{3}$. The obvious obstruction is that the characteristic curves can become linked in $R^{3}$.

We shall need the operation of the connected sum of two manifolds: $M=M_{1} \# M_{2}$. One constructs $M$ by deleting spherical balls from $M_{1}$ and $M_{2}$ and then gluing the manifolds together along the boundaries. Obviously, $M \# S^{3} \cong M$, which can be represented as the attachment of a single 3-cell to the spherical boundary of the ball obtained from $M$. This operation introduces an abelian semi-group structure and any 3-fold invariant can be regarded as a representation of this semi-group.

A manifold is called simple, if it cannot be represented as a connected sum of two non-spherical manifolds. Any compact oriented 3-manifold possesses a unique expansion into a connected sum of simple manifolds.

By performing a Heegaard splitting, a manifold is constructed out of two handlebodies joined by some homeomorphism of their boundaries. Such a homeomorphism can be continued into small neighborhoods of the boundaries. It means that the characteristic 
curves can submerge a bit into the inside of $H$. If $n_{i j}=\left(\alpha_{i}, \gamma_{j}\right)$ is an intersection number of two cycles on the boundary, then $\gamma_{j}$ will have after the deformation the same number as a linking coefficient with $\alpha_{i}$. The linking coefficient of two loops in $R^{3}$ is equal, by definition, to the intersection number of the first with a disk spanned by the second.

If $\alpha_{i}$ and $\gamma_{j}$ are linked, the corresponding 1-cell, $\sigma_{i}^{1}$, enters the boundary of the corresponding 2-cell, $\sigma_{j}^{2}: \sigma_{i}^{1} \in \partial \sigma_{j}^{2}$. And vice versa for co-boundaries: $\sigma_{j}^{2} \in \delta \sigma_{i}^{1}$. The boundary of a 2-cell defines a natural cyclic order of 1-cells belonging to it. A peculiarity of the dimension 3 is that 2-cells forming a co-boundary of a 1-cell are naturally ordered as well. It is a cyclic order of dual 1-cells forming a boundary of a dual 2-cell.

\subsection{Reshetikhin-Turaev functor}

The quantized function $(\mathrm{QF})$ algebra $\mathcal{F}_{q}(S L(n))$ is dual to the QUE algebra $\mathcal{U}_{q}(s l(n))$, therefore the topological basis of $\mathcal{F}_{q}(S L(n))$ is given by the matrix elements of irreducible representations of $\mathcal{U}_{q}(s l(n))$. For example, the $2 \times 2$ matrix realization of $S L_{q}(2)$ given in Eqs. (2.5) and (2.6) exactly corresponds to the matrix elements of the 2-dimensional irreducible representation of $\mathcal{U}_{q}(\operatorname{sl}(2))$. In this paper, we shall deal with real forms of $\mathcal{U}_{q}(\operatorname{sl}(n, C))$ with respect to some $*$-structures, whose existence is always assumed.

The discussion in the previous section suggests that we can construct $\mathrm{qQCD}_{3}$ with help of the Reshetikhin-Turaev functor from the category of colored ribbon tangles ctang to the category of representation rings of $\mathcal{U}_{q}(s l(n))$, $\operatorname{rep}_{\mathcal{U}_{q}}$.

The basic geometric object is a tangle, which was defined in Ref. [9] as "a link of circles and segments in the 3-ball, where it is assumed that ends of segments lie on the boundary of the ball". One puts into correspondence to every tangle a linear operator, $f$, acting on a tensor product of modules associated with segments (which have therefore to be oriented).

$$
f: V_{i_{1}} \otimes \ldots \otimes V_{i_{n}} \rightarrow V_{j_{1}} \otimes \ldots \otimes V_{j_{k}}
$$

or, graphically,

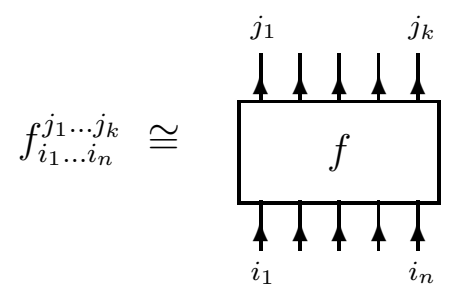

where $j_{1}, \ldots, j_{k}$ and $i_{1}, \ldots, i_{n}$ are some indices numerating modules. The simplest example 
is the identity operator represented by a single segment:

$$
\left.\delta_{\alpha, \beta} \cong\right|_{\beta} ^{\alpha}
$$

All modules considered in this paper are assumed to be irreducible. We shall draw linear operators acting on them as small boxes (coupons) with labels inside. The elementary building blocks are the matrix elements

$$
D_{\alpha, \beta}^{i}(a) \cong \sum_{i, \beta}^{\alpha}
$$

where $a$ is an element of $\mathcal{U}_{q}$. The arrows show a direction of the action of an operator. We use the Greek letters to numerate basis vectors of irreducible modules and the Latin ones, to numerate the modules. They will often be omited.

$\mathcal{U}_{q}$ possesses several $*$-structures. We shall draw conjugate objects as

$$
D_{\alpha, \beta}^{i}\left(a^{*}\right) \cong \sum_{i, \alpha}^{\beta}=\sum_{i, \bar{\alpha}}^{\bar{\beta}}
$$

The last equality takes place for a real form of $\mathcal{U}_{q}$, where the $*$-structure matches basis vectors of a module: $\alpha \rightarrow \bar{\alpha}$.

The operators form an algebra $\mathcal{A}$. We can translate this property in pictures as

$$
\{a, b \in \mathcal{A} \rightarrow a b \in \mathcal{A}\} \cong\left\{\frac{a b}{a}\right.
$$




$$
\{\exists 1 \in \mathcal{A}: a 1=1 a=a, \forall a \in \mathcal{A}\} \cong\left\{\frac{\uparrow}{1 a}=\stackrel{\uparrow 1}{=}=\frac{1}{a}\right\}
$$

$\mathcal{A}$ is a ring, i.e., an Abelian group under some + operation, which we shall understand as a formal sum of pictures with the natural definition of the multiplication by an integer number.

To have a bi-algebra structure on $\mathcal{A}$, we need a co-multiplication $\Delta: V \rightarrow V \otimes V$ and a co-unit $\varepsilon$. We introduce $\Delta$ as

$$
\Delta(a) \cong \frac{\Delta(a)}{\Delta+1}=\sum_{i}
$$

In general, the last equality is simply a convenient pictorial representation and has to be given a precise meaning in every particular case.

In Eq. (2.19), the 3-valent vertices,
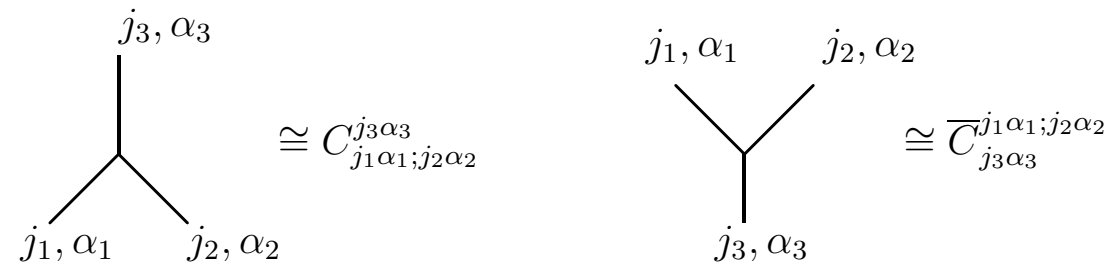

are the quantum Clebsch-Gordan coefficients $e_{\alpha_{1}}^{j_{1}} \otimes e_{\alpha_{2}}^{j_{2}}=\sum_{j_{3}, \alpha_{3}} C_{j_{1} \alpha_{1} j_{2} \alpha_{2}}^{j_{3} \alpha_{3}} e_{\alpha_{3}}^{j_{3}}\left(e_{\alpha}^{j}\right.$ is a basis of $\left.V_{j}\right)$. They obey the properties

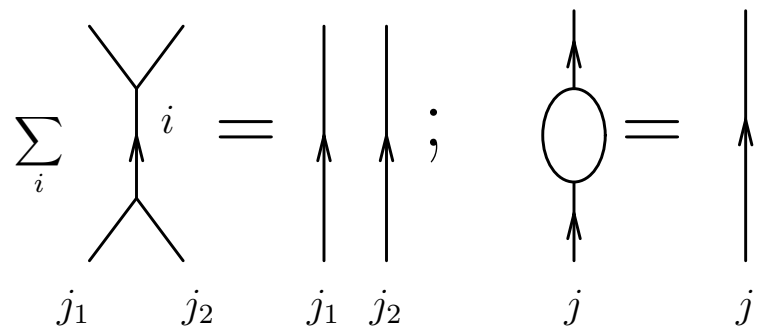

which simply means that they are elements of a unitary matrix connecting bases in $V$ and $V \otimes V$ : 


$$
\sum_{i, \beta} C_{j_{1} \alpha_{1} ; j_{2} \alpha_{2}}^{i \beta} \bar{C}_{i \beta}^{j_{1}^{\prime} \alpha_{1}^{\prime} ; j_{2}^{\prime} \alpha_{2}^{\prime}}=\delta_{j_{1}, j_{1}^{\prime}} \delta_{j_{2}, j_{2}^{\prime}} \delta_{\alpha_{1}, \alpha_{1}^{\prime}} \delta_{\alpha_{2}, \alpha_{2}^{\prime}}
$$

We can check the properties of the co-multiplication graphically

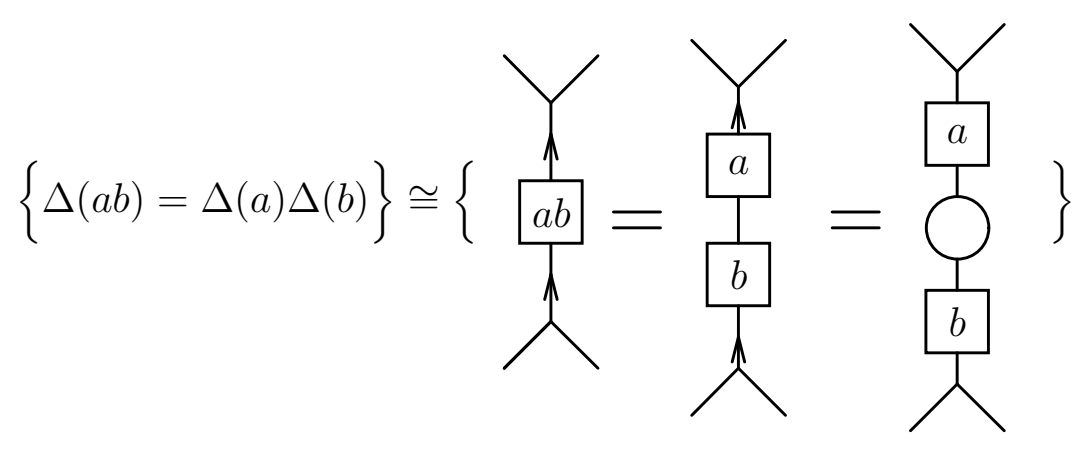

and

$$
\{\Delta(1)=1 \otimes 1\} \cong\{Y=||\}
$$

In these formulas, the sum over intermediate states is assumed. In what follows, we shall often omit the sum sign in pictures.

Thus, the co-associativity is coded in the properties of the Clebsch-Gordan coefficients.

The co-unit is a homomorphism to an abelian group associated with a field over which $\mathcal{A}$ is defined.

$$
\varepsilon(a b)=\varepsilon(a) \varepsilon(b) \quad \varepsilon(1)=1
$$

We shall connect the co-unit with a projection on the trivial representation of a quantum group. In other words, with the group integration.

To have the Hopf algebra structure on $\mathcal{A}$, we introduce an antipode map: $S: \mathcal{A} \rightarrow \mathcal{A}$ :

$$
S\left(\underset{\frac{1}{a}}{\uparrow}\right)=\stackrel{\substack{a \\ \frac{a^{*}}{4}}}{\uparrow}
$$


obeying

$$
\cdot(S \otimes i d) \circ \Delta=\cdot(i d \otimes S) \circ \Delta=1 \circ \varepsilon
$$

which looks graphically as

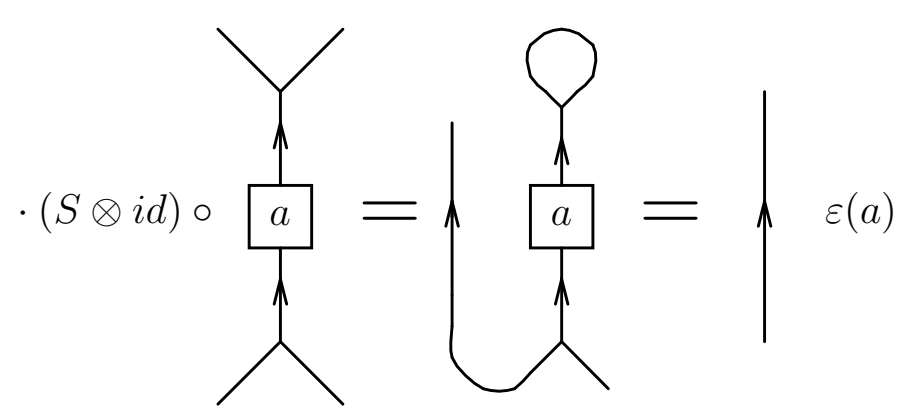

where $a$ is an arbitrary element of $\mathcal{A}$ and $\circ$ means a composition of operations. This property shows that the antipode can serve as a $q$-analog of the inverse. However, in general, $S^{2} \neq 1$.

The maps $V \otimes V \rightarrow C$ and $C \rightarrow V \otimes V$ are constructed with help of CGC:

$$
\bigcap_{j}:=\sqrt{d_{j}} \bigwedge_{j}^{0} ; \quad \underbrace{j}_{j}:=\left.\sqrt{d_{j}}\right|_{0} ^{j}
$$

where $d_{j}$ is the quantum dimension of the module $V_{j}$. These objects become the ordinary $\delta$-functions in the $q \rightarrow 1$ limit.

The self-consistency requires that

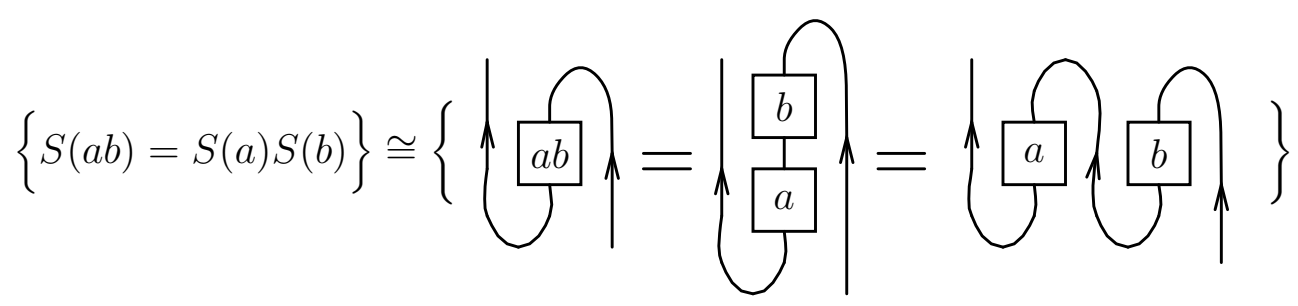


For this property to hold, it is important that

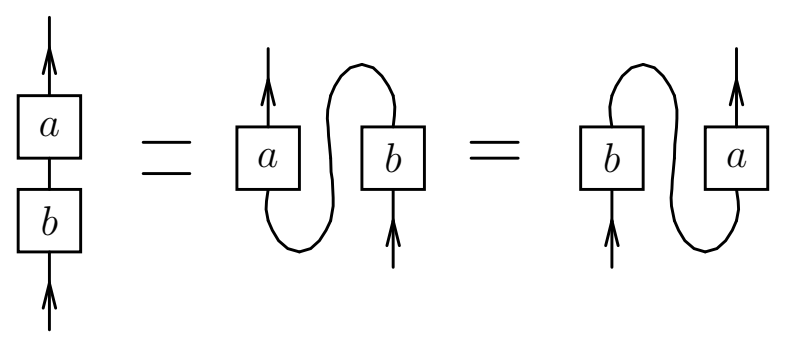

The other property of the antipode is $\Delta \circ S a=\tau \circ(S \otimes S) \circ \Delta a$. It can be checked graphically:

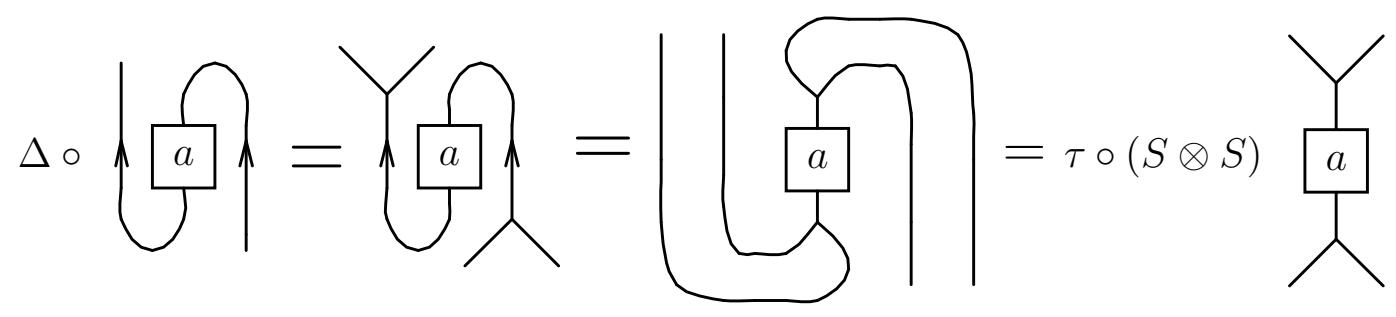

where $\tau$ is the flip operator: $V_{j_{1}} \otimes V_{j_{2}} \stackrel{\tau}{\longrightarrow} V_{j_{2}} \otimes V_{j_{1}}$.

Having equipped $\mathcal{A}$ with an $R$-matrix, we obtain a quasi-triangular Hopf algebra $(\mathcal{A}, R)$. The $R$-matrix obeys the Yang-Baxter equation (2.9). In our context, it will be more convenient to consider the $\check{R}$-matrix

$$
\check{R}:=\tau \circ R
$$

which can be represented graphically as

$$
\left\{\check{R}=\tau \circ \sum_{i} \alpha_{i} \otimes \beta_{i}\right\} \cong \searrow
$$

It is invertible

$$
\left\{\check{R}^{-1}=\tau \circ \sum_{i} \beta_{i} \otimes S\left(\alpha_{i}\right)\right\} \cong\{\searrow \backslash \backslash
$$

A Hopf algebra is called triangular if $\check{R}^{2}=1$. 
The standard definition of the $\check{R}$-matrix requires that the following properties hold

$$
\begin{aligned}
& \left\{(\Delta \otimes i d) \check{R}=\check{R}_{12} \check{R}_{23}\right\} \cong\{(\Delta \otimes i d) \\
& \left\{(i d \otimes \Delta) \check{R}=\check{R}_{12} \check{R}_{23}\right\} \cong\{(i d \otimes \Delta)
\end{aligned}
$$

along with the general form of Eq. (2.8)

$$
\left\{\check{R}(\Delta a) \check{R}^{-1}=\Delta a, \forall a \in \mathcal{A}\right\} \cong\{\stackrel{a}{a}=
$$

These equations are equivalent to the Yang-Baxter one

$$
\left\{\check{R}_{12} \check{R}_{23} \check{R}_{12}=\check{R}_{23} \check{R}_{12} \check{R}_{23}\right\} \cong\{
$$

The standard relations including the antipode are

$$
\begin{aligned}
& \left\{(S \otimes i d) \check{R}=\check{R}^{-1}\right\} \cong\{(S \otimes i d) \backslash= \\
& \left\{(i d \otimes S) \check{R}^{-1}=\check{R}\right\} \cong\{(i d \otimes S) \backslash /
\end{aligned}
$$

and those with the co-unit are

$$
\{(\varepsilon \otimes i d) \check{R}=(i d \otimes \varepsilon) \check{R}=1\} \cong\left\{\searrow_{j}=\searrow_{0}=\searrow_{j}\right\}
$$


The standard way to introduce the ribbon Hopf algebra structure on $\mathcal{A}$ is to bring forward the element $u \in \mathcal{A}$ defined as [9]

$$
\left\{u:=\sum_{i} S\left(\beta_{i}\right) \alpha_{i}\right\} \cong
$$

The element $v^{2}=u S(u)$ lies in the center of $\mathcal{A}$.

A ribbon Hopf algebra $\mathcal{U}_{q}=(\mathcal{A}, \check{R}, v)$ is a quasi-triangular Hopf algebra $(\mathcal{A}, \check{R})$ equipped with a central invertible element $v \in \mathcal{A}$,

$$
v:=v^{-1}:=0
$$

obeying the properties: $\varepsilon(v)=1$,

$$
\left\{u S(u)=v^{2}\right\} \cong\{u \circ
$$


The element

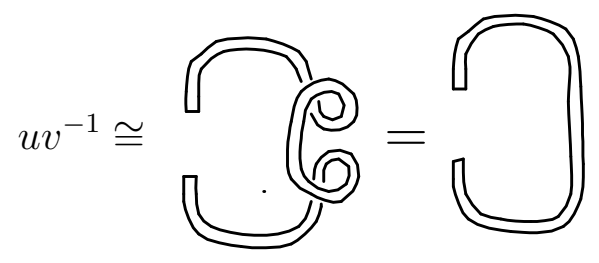

allows for defining the q-trace of an operator

$$
\mathrm{qtr}(a):=\operatorname{tr}\left(a u v^{-1}\right) \cong
$$

In the tensor square of spaces it takes the form

$$
\left\{\operatorname{tr}\left[a \otimes b \circ \Delta\left(u v^{-1}\right)\right]=\operatorname{qtr}(a) \operatorname{qtr}(b)\right\} \cong\{
$$

Following Reshetikhin and Turaev, we shall call this operation the closing of a tangle.

The quantum dimension of a module, $V_{j}$, is, by definition, the q-trace of the identity operator:

$$
d_{j}:=\operatorname{qtr}\left(1_{V^{j}}\right) \cong(
$$

\subsection{Algebra of fields and gauge invariance}

In Section 2.2 we have described a Heegaard diagram as a handlebody with a given system of $\alpha$-cycles and characteristic curves on its boundary. Every $\alpha$-cycle span a disk $D$ in a handlebody $H$. The disk can be thickened to a plate $P$. In this way we obtain a collection of disjoint plates in $H\left(P_{i} \cap P_{j}=\varnothing\right.$, if $\left.i \neq j\right)$. Each plate corresponds to a 1-cell of a base cell complex $C$ from which the Heegaard diagram has been read off. By detaching 
the plates, $H$ reassembles into a collection of 3-balls $\{B\}$, each corresponding to a 0 -cell of $C$.

Definition 1: We construct $\mathrm{qQCD}_{3}$ functor in the following way:

1. A gauge variable taking values in a ribbon QUE algebra $\mathcal{U}_{q}$ is put into correspondence to each plate:

$$
P_{k} \longrightarrow a_{k} \in \mathcal{U}_{q}
$$

The variables attached to different plates are distinct elements of $\mathcal{U}_{q}$, hence their matrix elements are co-commutative.

2. All the characteristic curves are colored with irreducible finite-dimensional representations of $\mathcal{U}_{q}$.

3. If on a boundary of the $k$-th plate there are $n_{k}$ disjoint cuts of the characteristic curves colored with representations $j_{1}, j_{2}, \ldots, j_{n_{k}}$, we construct a gauge field tangle by repeatedly applying the co-multiplication:

$$
F_{k}=\Delta^{n_{k}-1}\left(a_{k}\right): V_{j_{1}} \otimes \ldots \otimes V_{j_{n_{k}}} \rightarrow V_{j_{1}} \otimes \ldots \otimes V_{j_{n_{k}}}
$$

or, graphically,

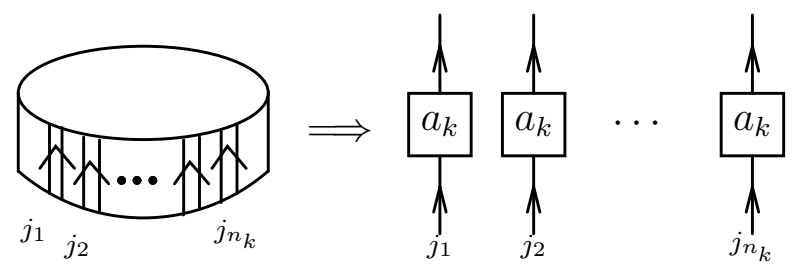

One has to respect the cyclic order and mutual orientations of the cuts. A reversion of an orientation of a cut corresponds to the conjugation(开) of the corresponding matrix element.

4. One puts into correspondence to each ball $B_{i} \in\{B\}$ carrying a pattern of the characteristic curves on its boundary a vertex tangle by using the Reshetikhin-Turaev functor ctang $\rightarrow \operatorname{rep}_{\mathcal{U}_{q}}$.

5. In the end, the pieces are attached together. For it, one embeds the handlebody into $R^{3}$ in such a way that the cuts of the characteristic curves on boundaries of the plates project to distinct points on the $(x, y)$ plane and onto disjoint segments on the $(x, z)$ plane. Then one can use the $(x, z)$ projection of the vertex tangles to complete the construction in terms of elements of a ribbon Hopf algebra as was depicted in the previous section. A result is a functional taking values in $C$.

\footnotetext{
$\dagger$ ) with respect to some fixed $*$-structure.
} 


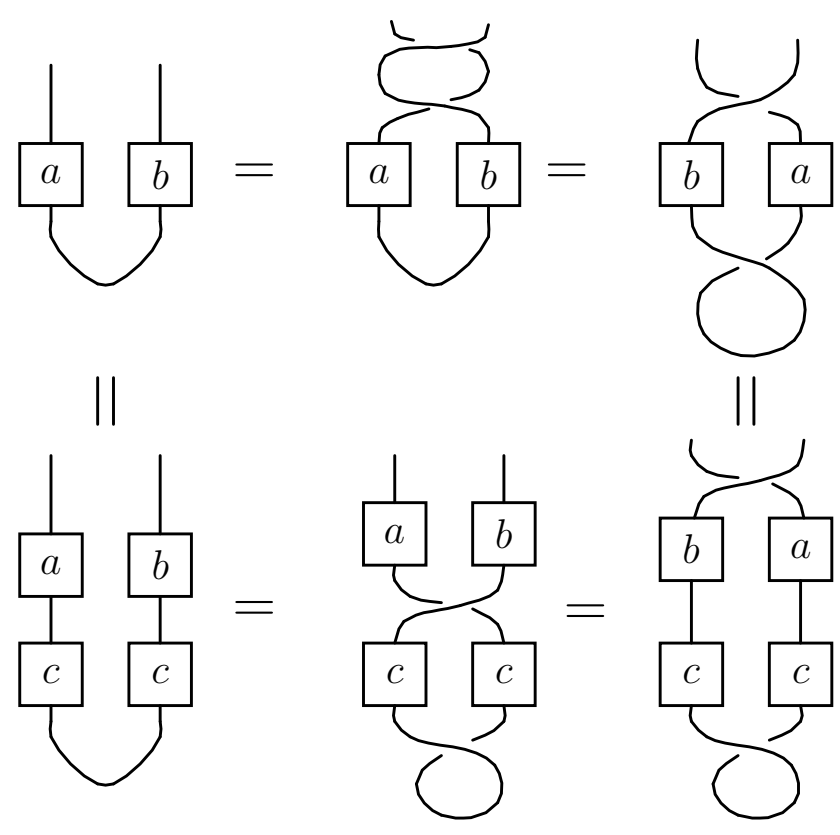

Figure 1: An illustration of the non-cocommutativity of fields adjacent via a vertex.

Remarks: 1) Let us notice that the initial data are colorings and directions of the characteristic curves as well as $\mathcal{U}_{q}$ elements attached to the plates.

2) One can sum over all the colorings with arbitrary weights as in Eq. (2.4). As we are restricted to real forms of $\mathcal{U}_{q}(\operatorname{sl}(n))$, the result has to be independent of the directions of the characteristic curves.

3) Modules appearing in different vertex tangles are independent. However, after the assemblage, all pieces are fit together and, permuting matrix elements adjacent via a vertex tangle, one has to deform the tangle, which means some effective non-cocommutativity. We shall dwell at this point later. Now, let us simply illustrate what may happen by the example shown in Figure 1.

4) Clearly, the construction gives different results for non-isotopic embeddings of $H$ into $R^{3}$. This lack of self-consistency will disappear after the integration over gauge fields (see the next section).

Let us now discuss the issue of gauge invariance within our framework. In the general settings of gauge theory the basic object is a fiber bundle over a base manifold. Gauge field performs a parallel transport of fibers and thus is interpreted as a $G$-connection in sections of the bundle. Gauge transformations act by automorphisms of the fibers. To make it explicit, one has to choose some $G$-basis at each point of the base. A quantity is gauge invariant if it is independent of a particular choice of the bases.

In LGT a base manifold is substituted by a finite cell complex. Therefore, instead of a fiber bundle, one has a tensor product of $G$-modules, one for each 0-cell in the complex. 
In our construction of $\mathrm{qQCD}_{3}, 0$-cells are associated with the vertex tangles. One chooses bases of the $\mathcal{U}_{q}$-modules for each tangle independently and then sandwiches the matrix elements between them. Thus, we can reformulate gauge invariance as the requirement of independence from particular choices of all the bases. As in the quantum case the notion of the group manifold is absent, one cannot translate a change of a frame into a group rotation. Although these changes can be given a matrix form, their status is quite different from the one of gauge fields. Therefore, we lose a contact with the explicit formula (2.1).

\subsection{Partition function}

We connect integration with the co-unit. By definition, it is a linear functional projecting onto the trivial representation. For the matrix elements, we have

$$
\left\{\int d a D^{j}(a)=\delta_{j, 0}\right\} \cong\left\{\varepsilon\left(\underset{\left.\right|_{j}}{\mid a}\right)=\delta_{j, 0}\right\}
$$

An integral of an arbitrary product of matrix elements can be reduced to the basic one

(2.51) by subsequently applying the tensor product decomposition with the ClebschGordan coefficients. For example, the orthogonality of matrix elements reads

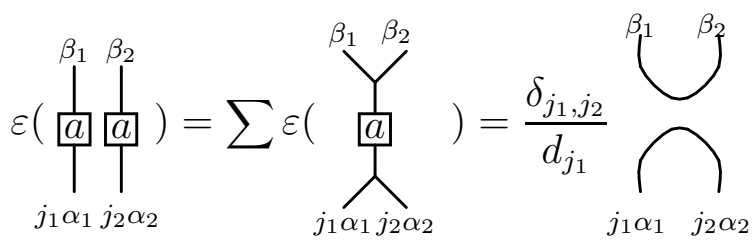

where $d_{j}$ is the q-dimension of a module $V_{j}$.

The selfconsistency of the definition can be easily checked

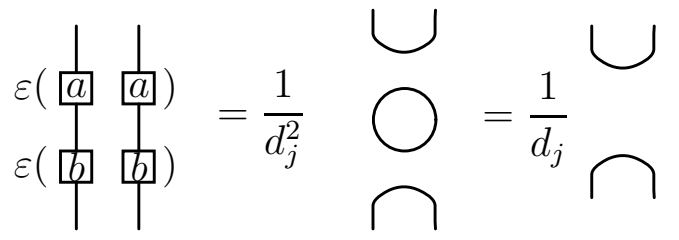


The main property of the Haar integral is the right/left invariance:

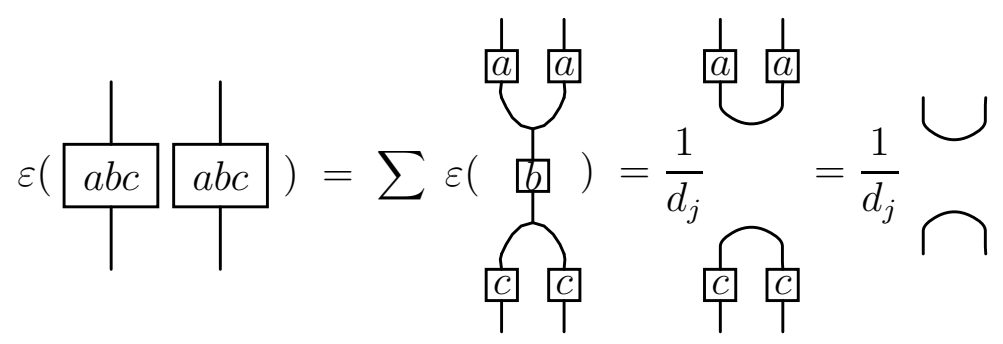

In the general case, the invariance easily follows from the properties of the Clebsch-Gordan coefficients and the antipode.

The integral should be used with some caution. For example, a reader has to be aware that

$$
\varepsilon(\underset{\mid a}{\mid a}) \mid \neq \varepsilon(\underset{|a|}{\mid a} \mid)
$$

and the r.h.s. of this formula makes no sense. Otherwise, one could easily arrive at contradictions. Expressions like $\int d x d y f(x, y)$ are inadequate in the quantum case. To exclude ambiguity, we shall always connect the integration with the linear operator acting on a tensor product of modules constructed with help of the co-multiplication:

DEFINITION 2:

$$
\int:=\varepsilon\left(\Delta^{n}()\right):\left(V_{1} \otimes \ldots \otimes V_{n+1} \stackrel{\Delta^{n}}{\longrightarrow} V_{1} \otimes \ldots \otimes V_{n+1}\right) \stackrel{\varepsilon}{\longrightarrow} C
$$

It can be calculated recursively:

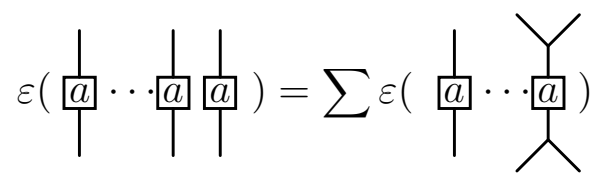

To complete our construction we need to specify a real form of $\mathcal{U}_{q}(\operatorname{sl}(n))$ with respect to some fixed $*$-structure. We are interested in 2 cases: (i) $\mathcal{U}_{q}(s u(n))$ which makes sense for real $q$ and (ii) $\mathcal{U}_{q}(s l(n, R))$ for $|q|=1$. The Hopf $*$-algebra $\mathcal{U}_{q}(s u(n))$ has been well investigated starting from the pioneering works of Woronowicz and Vaksman and Soibelman [3]. We need the following facts:

1. There is the one-to-one correspondence between finite dimensional irreducible representations of $\mathcal{U}_{q}(s u(n))$ and the classical algebra $\mathcal{U}(s u(n))$.

2. The representation ring of $\mathcal{U}_{q}(s u(n))$ spanned by matrix elements of finite-dimensional irreducible representations can be regarded as the $q$-deformation of the algebra of regular functions on $S U(N)$ (the quantum Peter-Weyl theorem). In particular, there exists a $q$-analog of the group $\delta$-function. 
3. There exists a q-analog of the Haar measure. The matrix elements are orthogonal with respect to it. An explicit representation of them can be given in terms of $q$-special functions. In this case the group integration is performed with help of the so-called Jackson integral from the $q$-special function theory.

Now, we are in a position to define the $\mathrm{qQCD}_{3}$ partition function. We shall denote the number of $k$-cells in a complex as $N_{k}$.

DEFINITION 3: We take the construction of the $\mathrm{qQCD}_{3}$ functor introduced in the previous section. Then

1. We color the characteristic curves with $\mathcal{U}_{q}(\operatorname{su}(n))$ irreps: $\gamma_{i} \rightarrow j_{i}, i=1, \ldots, N_{2}$.

2. We put into correspondence to every plate $P_{k}\left(k=1, \ldots, N_{1}\right)$ the integral

$$
P_{k} \longrightarrow \varepsilon\left(\Delta^{n_{k}-1}\left(a_{k}\right)\right), \quad a_{k} \in \mathcal{U}_{q}(s u(n))
$$

3. By applying Eqs. (2.51), (2.52) and (2.56) we obtain a collection of closed 3-valent ribbon graphs $\{\tau\}$, the number of which equals the number of 0 -cells in a base cell complex. By using the Reshetikhin-Turaev functor, we calculate the quantum invariant $\left(\right.$ 由, $J\left(\tau_{k}\right)$, for each connected component, $\tau_{k}$. Let us denote their product as

$$
Z_{j_{1} \ldots j_{N_{2}}}=\prod_{k=1}^{N_{0}} J\left(\tau_{k}\right)
$$

4. The partition function equals the sum over all colorings of the characteristic curves

$$
\mathcal{Z}_{\beta}=\sum_{\left\{j_{1} \ldots j_{N_{2}}\right\}} \prod_{k=1}^{N_{2}}\left(d_{j_{k}} e^{-\beta C_{j_{k}}}\right) Z_{j_{1} \ldots j_{N_{2}}}
$$

where $d_{j}$ is the quantum dimension and $C_{j}$ is a second Casimir eigenvalue.

Remarks: 1) If $q=1$, this definition reduces to the one given in Eqs. (2.3) and (2.4).

2) $\mathcal{Z}_{\beta}$ is a gauge invariant quantity in the sense described in the previous section. Indeed, as any vertex tangle after the integration gives a closed ribbon 3-valent graph, the choice of a basis attached to it is irrelevant.

3) After the integration, all non-isotopic embeddings of a handlebody $H$ into $R^{3}$ become equivalent and the consideration can be restricted to isotopies of the handlebody itself. 4) If one considers a cell complex dual to a simplicial one, the ribbon graph invariants $J\left(\tau_{k}\right)$ in Eq. (2.57) coincide with the quantum 6- $j$ symbols in the Racah-Wiegner normalization.

\footnotetext{
‡) Often called the generalized Jones polynomial.
} 


\subsection{The root of unity case}

For applications most interesting is the case when $q$ equals a primitive root of unity: $q^{\ell}=1$. Then $\mathcal{U}_{q}(s l(n))$ possesses the real form $\mathcal{U}_{q}(\operatorname{sl}(n, R))$. This case is rather complicated technically. One has to work with the restricted specialization $\mathcal{U}_{q}^{\text {res }}(\operatorname{sl}(n))$ of $\mathcal{U}_{q}(s l(n))$ and the issue of the duality between the QF and QUE algebras becomes quite subtle. Fortunately, one can go on with the notion of the modular Hopf algebra [9].

DEFINITION 4: Consider a ribbon Hopf algebra $(\mathcal{A}, \check{R}, v)$ equipped with a distinguished family $\left\{V_{j}\right\}_{j \in \mathcal{S}}$ of irreducible $\mathcal{A}$-modules indexed by a finite set $\mathcal{S}$ including the trivial representation $V_{0}$. $(\mathcal{A}, \check{R}, v)$ is called a modular Hopf algebra if the following requirements are fulfilled:

1. $\operatorname{qdim} V_{j} \neq 0, \forall j \in \mathcal{S}$.

2. The set $\left\{V_{j}\right\}_{j \in \mathcal{S}}$ is equipped with an involution $j \rightarrow j^{*}$ such that $V_{j^{*}}=V_{j}^{*}$ and $V_{j}^{* *}=V_{j}$.

3. For any sequence $j_{1}, \ldots, j_{n} \in \mathcal{S}$

$$
V_{j_{1}} \otimes V_{j_{2}} \otimes \ldots V_{j_{n}}=\bigoplus_{i \in \mathcal{S}} V_{i}^{\oplus m_{i}} \oplus I, \quad m_{i} \in N
$$

as $\mathcal{A}$-modules and for all $\mathcal{A}$-module endomorphisms, $f$, of the ideal $I$

$$
\operatorname{qtr}(f)=0
$$

4. Let $s_{i j}$ be the quantum invariant of the Hopf link, two components of which are colored with irreps $i$ and $j \in \mathcal{S}$

$$
s_{i j}=\operatorname{qtr}\left[\left(\frac{-b}{l_{j}}{ }^{i}\right]\right.
$$

then the matrix $\left(s_{i j}\right)_{i, j \in \mathcal{S}}$ is invertible.

Let us take the row of the inverse matrix $s^{-1}$ corresponding to the trivial representation $V_{0}$, then

$$
\sum_{j^{\prime} \in \mathcal{S}}\left(s^{-1}\right)_{0 j^{\prime}} s_{j^{\prime} j}=\delta_{0, j}
$$

We can consider Eq. (2.59) as an analog of the basic integral (2.51) with the obvious action of the co-multiplication:

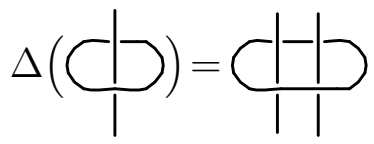


Owing to the third condition in the definition of the modular Hopf algebra, we find the following analog of the orthogonality of matrix elements

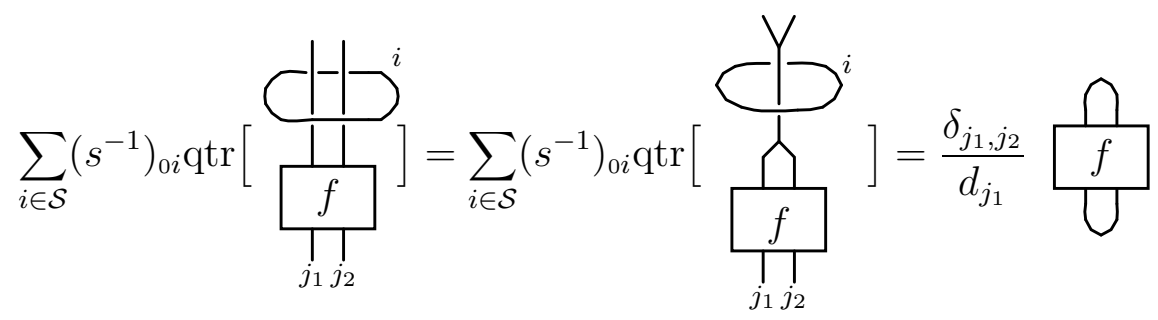

for any endomorphism $f: V \otimes V \rightarrow V \otimes V$. In these formulas, the $q$-trace is necessary to project out the ideal $I$.

An example of the modular Hopf algebra has been given by Reshetikhin and Turaev [9] in the $s l_{2}$ case. In Ref. [18] the notion of the quasi-modular Hopf algebra has been introduced by slightly weakening the irreducibility condition on the modules from $\left\{V_{j}\right\}_{j \in \mathcal{S}}$. It still leads to 3-manifold invariants of the Reshetikhin-Turaev type and therefore sufficient for our purposes as well. Turaev and Wenzl have constructed examples of quasi-modular Hopf algebras associated with $\mathcal{U}_{q}(\mathbf{g}), q^{\ell}=1$, for all $\mathbf{g}$ of the $A, B$ and $D$ types.

Thus, we define the $\mathrm{qQCD}_{3}$ partition function at a root of unity in the same way as in the previous section, using the given above definition of the integral. One can describe the quantity $Z_{j_{1} \ldots j_{N_{2}}}$ appearing in Eq. (2.57) as follows. We consider a Heegaard splitting $M=H \bigcup_{h} \widetilde{H}$. Let us continue a homomorphism $h$ into a small neighborhood of $\partial H$. In other words, $H \cap \widetilde{H}=M_{g}^{2} \times[0,1], \partial H=M_{g}^{2} \times 1$ and $\partial \widetilde{H}=M_{g}^{2} \times 0 ;\{\alpha\} \in \partial H$ and $\{\gamma\} \in \partial \widetilde{H}$. Then for any standard embedding of $H$ into $R^{3}$, the characteristic curves and the $\alpha$-cycles form a non-trivial link, $\mathcal{L}$. They are colored with two sets of representations $j_{1}, \ldots, j_{N_{2}} \in \mathcal{S}$ and $i_{1}, \ldots, i_{N_{1}} \in \mathcal{S}$. By using the Reshetikhin-Turaev functor, we calculate the quantum invariant of the link, $J_{j_{1} \ldots j_{N_{2}}}^{i_{1} \ldots i_{N_{1}}}(\mathcal{L})$, and sum over the colors of the $\alpha$-cycles with the weights $\left(s^{-1}\right)_{0 i}$ :

$$
Z_{j_{1} \ldots j_{N_{2}}}=\sum_{i_{1} \ldots i_{N_{1}} \in \mathcal{S}} \prod_{k=1}^{N_{1}}\left(s^{-1}\right)_{0 i_{k}} J_{j_{1} \ldots j_{N_{2}}}^{i_{1} \ldots i_{N_{1}}}(\mathcal{L})
$$

\subsection{The topological limit}

If in the root of unity case one chooses the Boltzmann weight coefficients equal to $v_{j}=\left(s^{-1}\right)_{0 j}$, one finds the partition function

$$
\mathcal{Z}_{0}(C)=\sum_{j_{1} \ldots j_{N_{2}} \in \mathcal{S}} \prod_{k=1}^{N_{2}} v_{j_{k}} Z_{j_{1} \ldots j_{N_{2}}}=\sum_{j_{1} \ldots j_{N_{2}} \in \mathcal{S}} \prod_{k=1}^{N_{2}} v_{j_{k}} \sum_{i_{1} \ldots i_{N_{1}} \in \mathcal{S}} \prod_{k=1}^{N_{1}} v_{i_{k}} J_{j_{1} \ldots j_{N_{2}}}^{i_{1} \ldots i_{N_{1}}}(\mathcal{L})
$$


where $N_{k}$ is the number of $k$-dimensional cells in a complex $C ; J_{j_{1} \ldots j_{N_{2}}}^{i_{1} \ldots i_{N_{1}}}(\mathcal{L})$ is the quantum invariant of a link $\mathcal{L}$ given by a Heegaard diagram associated to the complex $C$. Let us denote $\omega=\sum_{i \in \mathcal{S}} v_{i} d_{i}$.

THEOREM 1: $\mathcal{I}(\mathcal{M})=\mathcal{Z}_{0}(C) / \omega^{N_{0}+N_{3}-2}$ is a topological invariant of a manifold $\mathcal{M}$ represented by a complex $C . \mathcal{I}(\mathcal{M})$ is multiplicative with respect to the connected sum:

$$
\mathcal{I}(\mathcal{M})=\mathcal{I}\left(\mathcal{M}_{1}\right) \mathcal{I}\left(\mathcal{M}_{2}\right), \quad \text { if } \mathcal{M}=\mathcal{M}_{1} \# \mathcal{M}_{2}
$$

and $\mathcal{I}\left(S^{3}\right)=1$.

PRoof The Heegaard splitting associated to a cell complex $C$ having $N_{k}$ cells in the $k$ 'th dimension gives a handlebody, $H_{g}$, of the genus $g=N_{1}-N_{0}+1$. Let us fix $g$ independent $\alpha$-cycles of the Heegaard diagram and take the corresponding integrals (i.e., sums over $i$ 's in Eq. (2.63)) firstly. By applying the CGC decomposition and then using the orthogonality (2.61), we deform the set of the characteristic curves in the link $\mathcal{L}$ into some 3 -valent ribbon graph $\mathcal{G}$. Every application of Eq. (2.61) distroys a handle of $H_{g}$. Therefore, having taken the $g$ integrals, we obtain a spherical ribbon 3 -valent graph $\mathcal{G}$ plus a collection of $N_{1}-g=N_{0}-1$ disjoint unlinked loops corresponding to the rest of the $\alpha$-cycles. The integrals associated to them give $\omega^{N_{0}-1}$. Now, we can recover the intial configuration of the characteristic curves by restoring the $g$ integrals corresponding to the independent $\alpha$-cycles. In this way we obtain a cell decomposition of $\mathcal{M}$ with only one 0 -cell and every 1-cell corresponding to a generator of $\pi_{1}(\mathcal{M})$. This procedure is the direct analog of fixing an axial gauge in LGT.

The Heegaard splitting is obviously symmetric with respect to the Poincaré duality, therefore we can repeat the previous procedure with roles of the $\alpha$-cycles and the characteristic curves interchanged. In this way we fix a set of $g$ independent characteristic curves and pick up the factor $\omega^{N_{3}-1}$. Thus, we finish with some balanced presentation of $\pi_{1}(\mathcal{M})$.

To prove the topological invariance, we have to show that $\mathcal{I}(\mathcal{M})$ is not changed [i] by Dehn twists on contractible loops, [ii] by the cycle slide and [iii] by the stabilization.

i) Invariance under the Dehn twists on loops contractible inside $H_{g}$ is obvious. By taking the $g$ integrals, we cut all handles and always get the same 3 -valent ribbon graph $\mathcal{G}$.

ii) Invariance under the cycle slide follows from the analog of Haar measure invariance as illustrated in Figure 2.

iii) The stabilization consists in adding a handle to $H_{g}$ and extending a gluing homomorphism $h$ by the identity on its boundary. It amounts to the addition to $\mathcal{L}$ of one $\alpha$-cycle and one characteristic curve forming the Hopf link. Therefore, the integration associated to the new handle attaches the trivial representation to the new characteristic curve and it is unimportant how it is linked with the other $\alpha$-cycles.

\footnotetext{
$\S)$ or another one equivalent to it.
} 


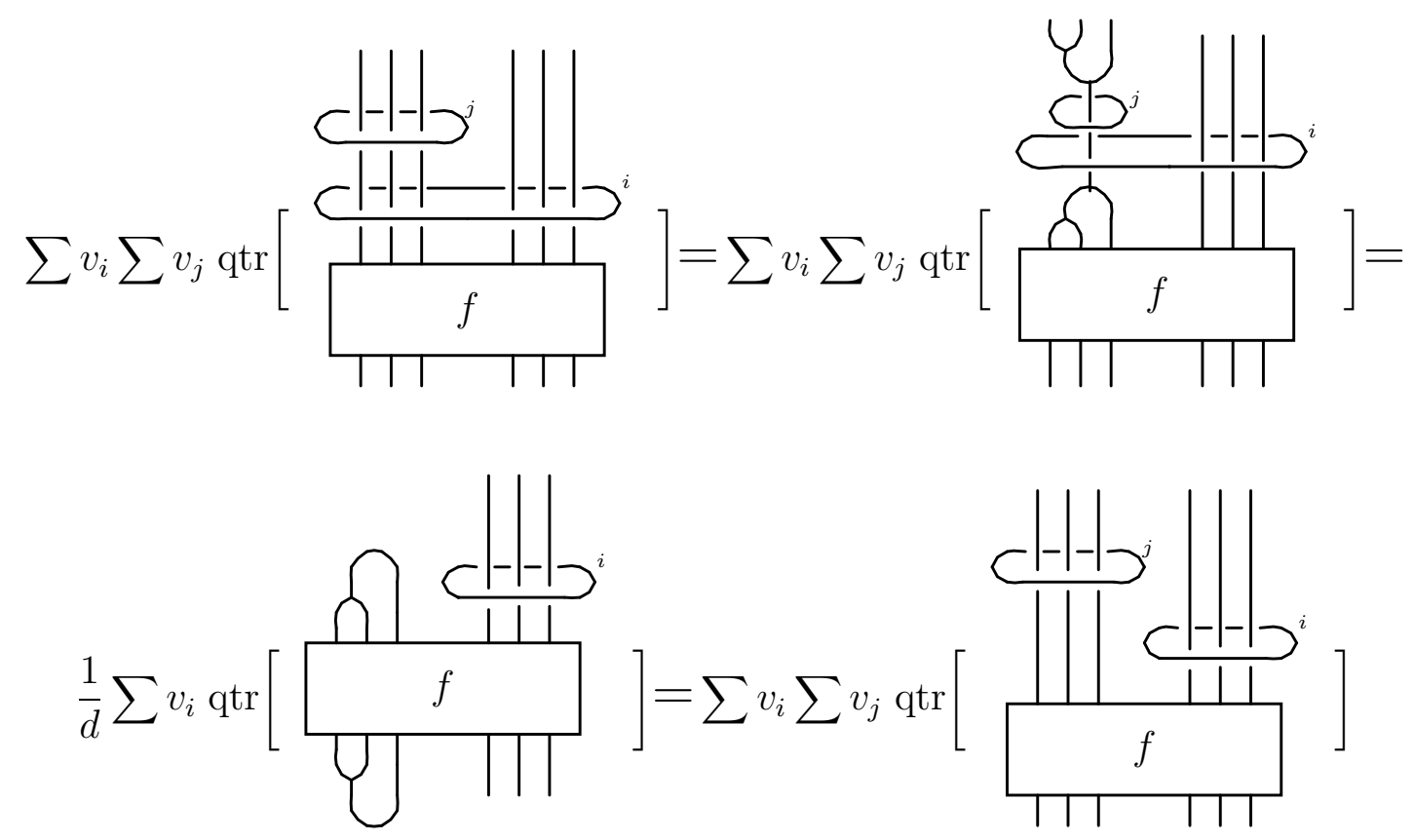

Figure 2: Invariance under the cycle slide.

To show the multiplicative nature of the invariant, let us choose such a cell decomposition of $\mathcal{M}=\mathcal{M}_{1} \# \mathcal{M}_{2}$ that a sphere dividing $\mathcal{M}_{1}$ and $\mathcal{M}_{2}$ consists of only one 0-cell and one 2-cell. Then the corresponding characteristic curve is linked with no $\alpha$-cycle and the corresponding link $\mathcal{L}$ in Eq. (2.63) has two connected components.

The normalization $\mathcal{I}\left(S^{3}\right)=1$ follows from the observation that the Hopf link corresponds to a genus 1 Heegaard splitting of the sphere.

Remarks: 1) The meaning of the choice of the Boltzmann weight coefficients made in Eq. (2.63) is clear. They corresponds to the $\delta$-function weights. Therefore, $\mathcal{Z}_{0}(\mathcal{M})$ can be regarded as a generalization of Eq. (2.10). In contrast to the finite group partition function, the $q$-deformed model is obviously self-dual with respect to the Poincaré duality of 3 -folds.

2) Let us consider a simplicial complex $C^{(s)}$. If we take in the expression (2.63) for the partition function $\mathcal{Z}_{0}\left(C^{(s)}\right)$ all the sums associated to triangles in $C^{(s)}$ prior to the others, then the answer is identical to the definition of the Turaev-Viro invariant. Indeed, for each triangle we find the tangle equivalent to the product of two $3 j$-symbols:

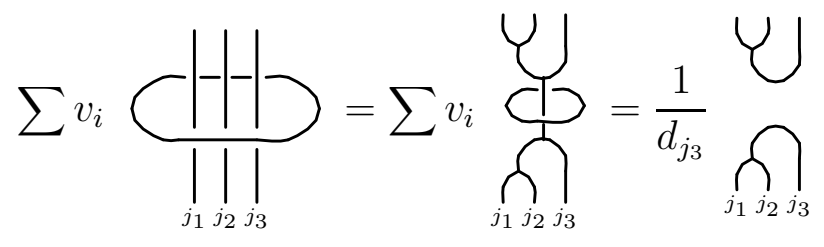


By closing all the tangles, we obtain a Racah-Wiegner $6 j$-symbol

$$
\left\{\begin{array}{lll}
j_{1} & j_{2} & j_{3} \\
j_{4} & j_{5} & j_{6}
\end{array}\right\}=\frac{1}{d_{j_{6}} \sqrt{d_{j_{2}} d_{j_{5}}}}
$$

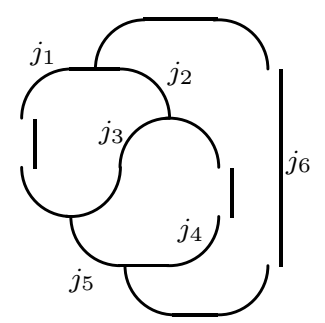

inside each tetrahedron of the simplicial complex. The indices, $j_{1}, \ldots, j_{N_{1}}$, are attached to 1-simplexes of $C^{(s)}$. Taking the sums over them we arrive at the Turaev-Viro state sum invariant [6]:

$$
\mathcal{I}_{0}\left(C^{(s)}\right)=\omega^{N_{1}-N_{2}+2} \sum_{\left\{j_{k} \in \mathcal{S}\right\}} \prod_{k=1}^{N_{1}} v_{j_{k}} \prod_{t=1}^{N_{3}}\left\{\begin{array}{ccc}
j_{t_{1}} & j_{t_{2}} & j_{t_{3}} \\
j_{t_{4}} & j_{t_{5}} & j_{t_{6}}
\end{array}\right\}
$$

where the 6 -tuple $\left(t_{1}, \ldots, t_{6}\right)$ denotes six edges of the $t$-th tetrahedron. Explicit expressions for $\left(s^{-1}\right)_{i j}$ in the $s l_{2}$ case are given in Ref. [9]. Thus, Eq. (2.63) can be regarded as a general definition of the Turaev-Viro invariant.

3) The expression for $\mathcal{Z}_{0}(\mathcal{M})$ given in Eq. (2.63) coincides with the Reshetikhin-Turaev construction of 3-fold invariants $I_{R T}$ via the surgery representation [9]. Therefore, $\mathcal{Z}_{0}(\mathcal{M})$ is automatically invariant under the Kirby calculus applied formally to the link $\mathcal{L}$. It means that, given a manifold $\mathcal{M}$, there exists another one $\mathcal{N}$ such that $\mathcal{I}(\mathcal{M})=I_{R T}(\mathcal{N})$. As $\mathcal{I}(\mathcal{M})=\left|I_{R T}(\mathcal{M})\right|^{2}$, we conclude that $\mathcal{N} \cong \mathcal{M} \# \overline{\mathcal{M}}(\overline{\mathcal{M}}$ is $\mathcal{M}$ with the opposite orientation). A simple illustration in the case of lens spaces can be found in Ref. [0].

\subsection{Bounded manifolds and links}

Every set of disjoint simple closed curves $\{\gamma\}$ on a handlebody $H$ determines a bounded 3-manifold $\mathcal{M}$ constructed by gluing plates to annular neighborhoods of the curves. It can be shown that every orientable bounded 3-manifold can be obtained in this way. The handlebody in this construction is a tubular neighborhood of a 1-skeleton of $\mathcal{M}$. Therefore we can straightforwardly apply the $\mathrm{qQCD}_{3}$ functor in the bounded case. For it, we [i] fix a system of $\alpha$-cycles on $\partial H$; [ii] color curves from $\{\gamma\}$ with $\mathcal{U}_{q}$ irreps; [iii] repeat steps 3,4 and 5 from Definition 1 without any modification. It suggest the following interpretation of our construction. A spine is a 2-dimensional polyhedron which can be embedded in some 3-manifold. Any 3-manifold with a boundary collapses to a spine. Let us delete a ball from every 3 -cell of a closed complex $C$. In such a way we obtain a bounded manifold which collapses to a 2 -skeleton $K_{2}$ of $C$. If $C$ is dual to a simplicial complex, $K_{2}$ is called a standard spine. Matveev has introduced two moves which relate all standard spines of the same manifold [19]. It can be easily shown that $\mathcal{I}(\mathcal{M})$ from the previous section is invariant under the Matveev moves. 
The definition of the $\mathrm{qQCD}_{3}$ functor uses an immersion of $K_{2}$ into $R^{3}$ and depends on it. It seems to be an intrinsic feature of q-deformed LGT rather than a defect of our presentation. Only gauge invariant singlet quantities (the partition function, for example) are independent of a way $K_{2}$ is immersed into $R^{3}$.

One of the advantages of our presentation of $\mathrm{qQCD}_{3}$ is a relative simplicity of introducing Wilson loops in it. In classical LGT, a loop average is defined as

$$
A\left(L_{1}, \ldots, L_{m}\right)=\frac{1}{\mathcal{Z}_{\beta}} \int_{G} \prod_{\ell} d g_{\ell} \prod_{f} W_{\beta}\left(h_{\partial f}\right) \prod_{i=1}^{m} \operatorname{tr}_{V_{j_{i}}}\left[h_{L_{i}}\right]
$$

where $\{L\}$ are $m$ closed curves embedded into a 1-skeleton $K^{1}$ of a complex $C$. We color the $i$-th curve with a representation $j_{i}$ of a gauge group $G$. The holonomy $h_{L}$ is defined in Eq. (2.2).

In the $q$-deformed case, we have to specify a link formed by the collection of curves in a manifold $\mathcal{M} \cong C$. For it we represent the curves $\{L\}$ by a set of disjoint ribbon loops on a boundary $\partial H^{\prime}$ of a handlebody $H^{\prime} \subset H$ (as usual, $H^{\prime}$ and $H$ are tubular neigborhoods of $K^{1}$ and $H^{\prime}$ lies inside $\left.H: H \cap H^{\prime}=H^{\prime}, H \cup H^{\prime}=H\right)$. If it is not possible, then one has to take a finer subdivision of $\mathcal{M}$. In the case of links in $R^{3}$, it is a standard technical trick to realize a link as a system of disjoint loops on a handlebody embedded into $R^{3}$. And we simply use it as a definition.

We can apply the $\mathrm{qQCD}_{3}$ functor to such a composite handlebody without any additional modification. Loops from $\{L\}$ enters on equal footing with characteristic curves. One can repeat the same argument as in the partition function case to prove that an answer is independent of an embedding of $H$ in $R^{3}$. However, it does not mean that the $q$-deformation of Eq. (2.67) gives no non-trivial knot invariant. Let us consider a link in $R^{3}$. There has to exist a trivial embedding such that characteristic curves of a Heegaard diagram lying on $\partial H$ are unlinked and contractible in $R^{3} \backslash H$. Therefore the sums over their colors disjoin $\alpha$-cycles on $\partial H$ and the link of curves $\{L\}$ on $\partial H^{\prime}$ (in other words, cut handles of the complementary handlebody $S^{3} \backslash H$ ). What remains is exactly the Jones polynomial associated to the link $\{L\}$.

The comprehensive treatment of quantum invariants of links and 3-valent graphs in 3-manifolds can be found in Ref. [20].

\section{$3 \quad \mathrm{qQCD}_{2}$}

We define $\mathrm{qQCD}_{2}$ functor by applying the $\mathrm{qQCD}_{3}$ one to an embedding of an oriented 2-manifold $M_{g}^{2}$ in $R^{3}$. In the topological limit, we can consider the simplest cell decomposition of $M_{g}^{2}$ consisting of a single 2-cell and $2 g$ 1-cells. A tubular neigborhood of its 1 -skeleton is a handlebody $H$ of the genus $2 g$. The Heegaard diagram has only one characteristic curve. Each integral destroys a handle of $H$ and contributes a factor $1 / d_{j}$ to an 
answer. The calculation is reduced to a repeated application of Eq. (2.61) and one easily gets

$$
\mathcal{I}\left(M_{g}^{2}\right)=\omega^{2 g-1} \mathcal{Z}_{0}\left(M_{g}^{2}\right)=\omega^{2 g-1} \sum_{j \in \mathcal{S}} v_{j} Z_{j}=\sum_{j \in \mathcal{S}} v_{j}\left(\frac{d_{j}}{\omega}\right)^{1-2 g}
$$

Let us consider a concrete example of the quantum group $\mathcal{U}_{q}(s l(2))$, at $q=e^{\frac{2 \pi i}{k+2}}$. In this case, the set of modules in the definition of the modular Hopf algebra is given by the fusion ring $V_{j}\left(j=0, \frac{1}{2}, 1, \frac{3}{2}, \ldots, \frac{k}{2}\right)$ and $d_{j}=\frac{\sin \left(\frac{2 j+1}{k+2} \pi\right)}{\sin \frac{\pi}{k+2}} ; \omega=\frac{\sqrt{(k+2) / 2}}{\sin \frac{\pi}{k+2}} ; v_{j}=d_{j} / \omega$. We find

$$
\mathcal{I}\left(M_{g}^{2}\right)=\sum_{j=0, \frac{1}{2}, \ldots}^{k / 2}\left(\frac{2 \sin ^{2}\left(\frac{2 j+1}{k+2} \pi\right)}{k+2}\right)^{1-g}
$$

These are known as Verlinde's numbers. They are all integer and equal to the dimensions of spaces of conformal blocks in the WZW model on a genus $g$ Riemann surface.

If one starts with a more complicated cell decomposition of a Riemann surface, then one has simply apply the orthogonality relation (2.61) till all handles of $H$ are destroyed.

In two dimensions, local properties of $\mathrm{qQCD}_{2}$ functor can be formalized in a pure algebraic way. For it, let us cut from $M_{g}^{2}$ a piece which can be projected on a plane $R^{2}$. It gives a subdivision (triangulation, say) of some region on the plane. There is a natural cyclic order of edges incident to a vertex. Following Fock and Rosly [15], one introduces a ciliation at every vertex, i.e., breaks this order. Let us say that an edge $\ell_{1}$ goes after $\ell_{2}\left(\ell_{1}>\ell_{2}\right)$, if an anti-clockwise angle $\varphi\left(\ell_{1}\right)$ between the edge $\ell_{1}$ and the $x$-axis is bigger than an angle $\varphi\left(\ell_{2}\right)$ between $\ell_{2}$ and the $x$-axis: $\varphi\left(\ell_{1}\right)>\varphi\left(\ell_{2}\right)$. We assume that no edge is parallel to the $x$-axis, and orient edges in the $y$-direction. Say, put an arrow at an end having a bigger $y$ coordinate. Assuming that any two vertices are connected at most by one edge, we can numerate edges by ordered pairs of vertices $(i, j) \cong{ }_{j} \nearrow^{i}$.

Alekseev, Grosse and Schomerus have introduced the following algebra of gauge fields $U_{(i, j)}$ [13]:

i) If two edges have no common vertices, fields are co-commutative:

$$
\stackrel{1}{U}_{(i, j)} \stackrel{2}{U}_{(n, m)}=\stackrel{2}{U}_{(n, m)} \stackrel{1}{U}_{(i, j)}
$$

here $i, j, n$ and $m$ are all distinct.

ii) If two edges share a vertex, then

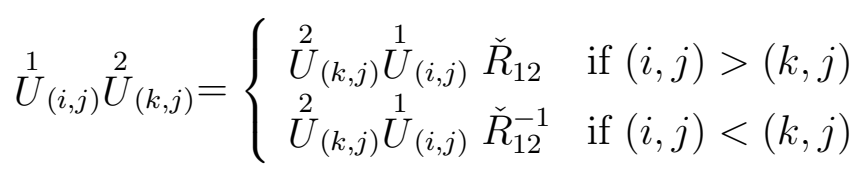


We picture these relations as

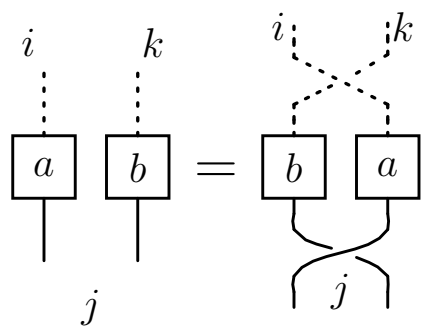

$$
\text { if }(i, j)>(k, j)
$$

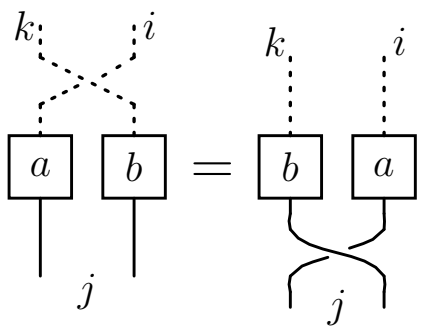

$$
\text { if }(i, j)<(k, j)
$$

We have drawn in solid lines $\mathcal{U}_{q}$-elements figuring in the AGS relations associated to the $j$-th vertex. All attached to other vertices are dashed.

iii) Fields attached to the same edge form a quasi-triangular Hopf algebra:

1. $R_{12}^{-1} \stackrel{1}{U}{ }_{(i, j)} \stackrel{2}{U}_{(i, j)} R_{12}=\stackrel{2}{=} \stackrel{1}{U} \stackrel{1}{U}_{(i, j)}$

2. $\cdot \stackrel{1}{U} \underset{(j, i)}{\stackrel{2}{U}}(i, j)=1$. This property is sometimes called the cancellation of a backtracking:

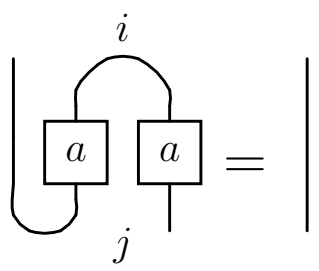

Remarks: 1) The properties [i] and [iii] are obviously agreed with our definition of $\mathrm{qQCD}_{2}$ (see, e.g., , the pictorial illustration in Figure 1, and the discussion preceding it). The relations [ii] follow from transformations of modules associated to vertex tangles. Of course, being made, such a move has to be compensated somewhere by its reciprocal for a whole construction to remain invariant.

2) As a closed surface can be projected onto $R^{2}$ only locally, one has to use gluing homomorphisms to assembly a Riemann surface out of flat pieces. These homomorphisms match the gauge field algebra relations on different pieces and have to be added in order to complete the construction.

3) The set of the AGS relations is distinguished by an observation that they generate a lattice Kac-Moody algebra in the sense of Ref. [21]. However, they do not constitute all possible symmetries of the $\mathrm{qQCD}_{2}$ functor. 


\section{Concluding remarks}

1. The first natural question to ask is whether the results of this paper could be generalized to higher dimensions. The answer is certainly "No"! The reason for it is that, in dimensions bigger than 3, there is no natural ordering of faces incident to an edge in a complex. It restricts the class of acceptable Hopf algebras to triangular ones. Then the corresponding construction essentially coincides with the classical Wilsonian LGT.

2. It is tempting to interpret the topological invariant considered in this paper as some suitable generalization of Eq. (2.10) and the construction of topological $\mathrm{qCDQ}_{3}$ as a generalization of $H^{1}(C, G)$. Unfortunately, we are able to say nothing constructive about it. However, in the 2-dimensional case, a notion of a quantum moduli space could presumably be formulated [15], which leaves some hope for the future.

3. We conjecture that $\mathrm{qQCD}_{3}$ with $\mathcal{U}_{q}(s u(n))$ gauge group possesses a continuum limit equivalent to a gauge theory whose action includes both Yang-Mills and ChernSimons terms. One could introduce a non-zero coupling constant in the root-of-unity case as well, which implies some deformation of Chern-Simons theory. A meaning of this procedure is absolutely unclear to us.

\section{Acknowledgments}

I thank V.Turaev for the fruitful discussion. This work was supported by the EEC program "Human Capital and Mobility" under the contract ERBCHBICT9941621.

\section{References}

[1] K.Wilson, Phys. Rev. D10 (1975) 2445.

[2] V.G.Drinfeld, "Quantum Groups", in Proc. ICM (1987) 798;

L.D.Faddeev, N.Reshetikhin and L.Takhtajan, Leningrad Math. J. 1 (1990) 193.

[3] S.L.Woronowicz, Commun. Math. Phys. 111 (1987) 613;

L.L.Vaksman and Ya.S.Soibelman, Func. Anal. Appl. 22 (1988) 170.

[4] D.V. Boulatov, Mod. Phys. Lett. A7 (1992) 1629.

[5] G.Ponzano and T. Regge, in Spectroscopic and group theoretical methods in physics, ed. F.Bloch (Amsterdam, 1968).

[6] V.G.Turaev and O.Y.Viro, Topology 31 (1992) 865;

V.G.Turaev, C.R. Acad. Sci. Paris 313 (1991) 395 and J. Diff. Geom. 36 (1992) 35. 
[7] D.V. Boulatov, Int. J. Mod. Phys. A8 (1993) 3139.

[8] D.V.Boulatov, "3D Gravity and Gauge Theories", in NATO Advanced Studies Institutes Series B, vol. 328, p. 39.

[9] N.Yu.Reshetikhin and V.G.Turaev, Commun. Math. Phys. 124 (1989) 307 and Invent. Math. 103 (1991) 547.

[10] E.Witten, Comm. Math. Phys. 121 (1989) 351 and Nucl. Phys. B311 (1988/89) 46.

[11] D.V. Boulatov, Mod. Phys. Lett. A8 (1993) 3491.

[12] A.Alekseev, St.-Peterburg Math. J. 6 (1994) 1.

[13] A.Alekseev, H.Grosse and V.Schomerus, Comm. Math. Phys. 172 (1995) 317.

[14] E.Buffenoir and P.Roche, Comm. Math. Phys. 170 (1995) 669.

[15] V.V.Fock and A.A.Rosly, "Poisson structure on moduli of flat connections on Riemann surfaces and r-matrix", preprint ITEP $72-92$ (1992).

[16] T.Kohno, Topology 31 (1992) 203.

[17] L.Crane, Com. Math. Phys. 135 (1991) 615.

[18] V.Turaev and H.Wenzl, Int. J. Math. 4 (1993) 323.

[19] S.V.Matveev, Math. USSR Izvestiya 31 (1988) 423.

[20] V.Turaev, Publ. Math. IHES 77 (1993) 121.

[21] A.Alekseev, L.Faddeev and Semenov-Tian-Shansky, Comm. Math. Phys. 149 (1992) 335. 